\title{
Rastreamento do câncer do colo do útero em Minas Gerais: avaliação a partir de dados do Sistema de Informação do Câncer do Colo do Útero (SISCOLO)
}

\author{
Cervical cancer tracking in Minas Gerais: assessment of \\ data from Cervical Cancer Information System (SISCOLO)
}

\author{
Camila Soares Lima Corrêa', Amanda de Souza Lima1', \\ Isabel Cristina Gonçalves Leite ${ }^{1,2,3}$, Luanna Couto Pereira4, \\ Mário Círio Nogueira ${ }^{2,3}$, Daniela de Almeida Pereira Duarte ${ }^{1}$, \\ Vívian Assis Fayer ${ }^{1}$, Maria Teresa Bustamante-Teixeira ${ }^{1,2,3}$
}

\begin{abstract}
Resumo
Objetivo: Avaliar os indicadores relacionados à oferta de exames citopatológicos do colo do útero, sua qualidade, frequência de alterações celulares e seguimento informado de lesões de alto grau, em Minas Gerais e suas macrorregiões de saúde. Métodos: Estudo descritivo com base em dados do Sistema de Informação do Câncer do Colo do Útero (SISCOLO), de 2006 a 2011. Resultados: A razão de exames citopatológicos do colo do útero em mulheres de 25 a 59 anos manteve-se estável, porém sem alcançar a meta estadual pactuada. Aproximadamente $75 \%$ dos exames foram realizados na população-alvo e houve progressiva redução na proporção de citopatológicos do colo do útero sem citologia anterior. Em média, $51,2 \%$ dos exames foram realizados no período de até um ano. Quanto à qualidade do exame, destaca-se o índice de positividade, categorizado como baixo durante todo o período analisado. Observou-se baixo percentual de seguimento informado no estado. Conclusão: Os resultados evidenciam a necessidade de aprimoramento do programa de rastreamento em relação à oferta e qualidade do exame, bem como ao direcionamento correto quanto à faixa etária e à periodicidade, visando garantir o acesso da população-alvo e o encaminhamento para a investigação diagnóstica e tratamento das lesões precursoras, quando indicado.
\end{abstract}

Palavras-chave: neoplasias do colo do útero; programas de rastreamento; Saúde da Mulher; Sistemas de Informação.

\footnotetext{
Abstract

Objective: To evaluate the indicators related to cervical cytology, quality, frequency of cellular changes and informed follow-up of high-grade lesions in Minas Gerais and its macro-regions of health. Methods: a descriptive study based on data from the Cervical Cancer Information System (SISCOLO) from 2006 to 2011. Results: The ratio of cervical cytopathologic exams in women aged 25 to 59 years remained stable, but did not reach the goal set by the state. Approximately $75 \%$ of the exams were performed in the target population and there was a progressive reduction in the proportion of cervical cytopathologists without previous cytology.

1Programa de Pós-graduação em Saúde Coletiva, Universidade Federal de Juiz de Fora (UFJF) - Juiz de Fora (MG), Brasil.

2Departamento de Saúde Coletiva, Faculdade de Medicina, Universidade Federal de Juiz de Fora (UFJF) - Juiz de Fora (MG), Brasil.

${ }_{3}^{3}$ Programa de Pós-graduação em Saúde, Faculdade de Medicina, Universidade Federal de Juiz de Fora (UFJF) - Juiz de Fora (MG), Brasil.

${ }^{4}$ Núcleo de Assessoria, Treinamentos e Estudos em Saúde (NATES), Universidade Federal de Juiz de Fora (UFJF) - Juiz de Fora (MG), Brasil.

Trabalho realizado no Núcleo de Assessoria, Treinamento e Estudos em Saúde (NATES), Universidade Federal de Juiz de Fora (UFJF) - Juiz de Fora (MG), Brasil.

Endereço para correspondência: Camila Soares Lima Corrêa, Rua Marechal Deodoro, 1019/401 - Centro - CEP: 36015-460 - Juiz de Fora (MG), Brasil -

Email: camilaslcorrea@hotmail.com

Fonte de financiamento: Fundação de Amparo à Pesquisa do Estado de Minas Gerais (FAPEMIG) - CDS-APQ 03630-12. As autoras Isabel Cristina Gonçalves Leite e Maria Teresa Bustamante-Teixeira são bolsistas de produtividade em pesquisa do Conselho Nacional de Desenvolvimento Científico e Tecnológico (CNPq).

Conflito de interesses: nada a declarar.
} 
On average, $51.2 \%$ of the exams were performed within a year. Regarding the quality of the exam, the positivity index was categorized as low throughout the analyzed period. We observed a low percentage of follow-up reported in the state. Conclusion: The results evidenced the need to improve the tracking program regarding the quality of the exam, as well as the correct orientation regarding the age group and the periodicity, aiming to guarantee the target population's access and referral for diagnostic investigation and treatment of precursor lesions, when indicated.

Keywords: uterine cervival neoplasms; mass screening; Women's Health; Information Systems.

\section{INTRODUÇÃO}

O câncer do colo do útero (CCU) é o quarto tipo de câncer mais comum em mulheres no mundo ${ }^{1}$. Em 2012, as estimativas apontaram 528 mil casos novos, dos quais cerca de $80 \%$ ocorreram em regiões menos desenvolvidas ${ }^{2}$. Excluindo o câncer de pele não melanoma, é o terceiro tipo de câncer mais frequente nas mulheres brasileiras. Na região Norte, é o mais incidente, e, nas regiões Centro-Oeste e Nordeste, ele ocupa a segunda posição ${ }^{3}$. Quanto à mortalidade, apesar da queda observada no país nos últimos anos, ela não ocorre em todas as regiões, como no interior do Norte e Nordeste 4 .

O rastreamento realizado por meio do exame citopatológico do colo do útero é considerado a principal estratégia para a detecção precoce do CCU, sendo reconhecido mundialmente como seguro e eficiente. O objetivo fundamental é detectar e tratar precocemente as lesões precursoras antes da sua evolução para a doença invasiva ${ }^{5}$. Nesse sentido, com uma cobertura da população-alvo de, no mínimo, $80 \%$ e acesso à confirmação diagnóstica e ao tratamento adequado, é possível reduzir em até $90 \%$ a incidência do câncer cervical invasivo ${ }^{6}$.

O Ministério da Saúde recomenda o rastreamento citopatológico em mulheres de 25 a 64 anos, a cada três anos após dois exames com resultados negativos realizados anualmente ${ }^{5}$. Ao contrário de países desenvolvidos, onde há recrutamento ativo da população-alvo para o rastreamento, no Brasil o rastreamento ainda é oportunístico, com procura ocasional e espontânea dos serviços de saúde determinada por razões diversas que não o rastreamento do $\mathrm{CCU}^{7}$. O rastreamento oportunístico, além de ser menos efetivo no impacto sobre a mortalidade, é mais oneroso para o sistema de saúde ${ }^{8}$.

O controle da doença é assumido como uma prioridade nacional ${ }^{5}$. Entre as principais ações nesse sentido, destacam-se a instituição pelo Ministério da Saúde, em 1998, do Programa Nacional de Combate ao Câncer do Colo do Útero e a criação do Sistema de Informação do Câncer do Colo do Útero (SISCOLO). Esse sistema foi implantado oficialmente no país pela Portaria/SAS/MS no 408, de 30 de julho de 1999, e visa viabilizar o gerenciamento das ações de rastreamento do CCU. Posteriormente, foi aperfeiçoado, e, em 2006, foi implantada uma nova versão (4.0) pela Portaria/SAS/MS n 287 , de 24 de abril de 2006. Em 2005, foi instituída a Política Nacional de Atenção Oncológica, e, em 2006, a importância da detecção precoce do
CCU foi reafirmada no Pacto pela Saúde, por meio da inclusão de indicadores específicos na pactuação de metas com Estados e municípios ${ }^{5}$.

Minas Gerais se caracteriza pela grande extensão e diversidade regional, sendo considerada uma representação da estrutura regional brasileira, menos desenvolvida ao norte/nordeste e mais desenvolvida ao sul ${ }^{9}$. Assim, torna-se relevante avaliar os indicadores relacionados ao rastreamento do CCU, com foco estadual, que permita monitorar indicadores pactuados e identificar diferenças regionais, podendo contribuir para o planejamento de ações mais resolutivas.

O presente estudo visa avaliar as ações de rastreamento com base em dados do SISCOLO em Minas Gerais e suas macrorregiões de saúde a partir da análise de indicadores relacionados às características da oferta de exames citopatológicos, à qualidade do exame, à ocorrência de alterações celulares nos exames e ao seguimento informado dos casos de lesão de alto grau.

\section{MÉTODOS}

Trata-se de um estudo descritivo com base em dados secundários do SISCOLO que analisa os indicadores, em Minas Gerais e suas macrorregiões de saúde, no período de 2006 a 2011. O Estado possui 853 municípios, 77 microrregiões e 13 macrorregiões de saúde, caracterizadas pela grande disparidade socioeconômica, as quais foram as unidades de análise do estudo.

Os dados foram extraídos do sítio eletrônico do Departamento de Informática do Sistema Único de Saúde (DATASUS) ${ }^{10}$, nos meses de abril e maio de 2016, e a descrição dos indicadores calculados e as respectivas fontes dos dados encontram-se no Quadro 1. A oferta do exame foi avaliada pelo indicador razão de exames citopatológicos em mulheres de 25 a 59 anos, utilizado no Pacto pela Saúde ${ }^{11}$ cujas metas estão disponíveis, a partir de 2007, no Sistema de Informação do Pacto pela Saúde (SISPACTO). Apesar de a recomendação do INCA quanto à faixa etária da população-alvo de rastreamento do CCU ter sido atualizada no ano de 2011 para 25 a 64 anos, optou-se por analisar a faixa etária de 25 a 59 anos, seguindo a recomendação vigente naquele período ${ }^{12}$.

A oferta de exames foi caracterizada pela faixa etária e periodicidade da realização. Por meio desses indicadores, é 
Quadro 1. Descrição dos indicadores calculados e respectivas fontes dos dados

\begin{tabular}{|c|c|c|}
\hline Indicador & Cálculo & $\begin{array}{c}\text { Fonte dos } \\
\text { dados }\end{array}$ \\
\hline $\begin{array}{l}\text { Razão de exames citopatológicos do colo do útero em } \\
\text { mulheres de } 25 \text { a } 59 \text { anos }\end{array}$ & $\begin{array}{l}\text { Número total de exames citopatológico em mulheres de } 25 \text { a } \\
59 \text { anos / população feminina nessa faixa etária }\end{array}$ & $\begin{array}{l}\text { SISCOLO } \\
\text { IBGE }^{\mathrm{b}}\end{array}$ \\
\hline $\begin{array}{l}\text { Proporção de exames citopatológicos por faixa etária } \\
\text { (<25 anos; } 25-59 \text { anos; } \geq 60 \text { anos) }\end{array}$ & $\begin{array}{l}\text { Número de exames citopatológicos em mulheres que relataram } \\
\text { que já se submeteram ao exame anterior x } 100 \text { / número } \\
\text { total de exames de rastreamento - o número de exames em } \\
\text { mulheres que não souberam informar se já haviam realizado o } \\
\text { procedimento }\end{array}$ & SISCOLO $^{\mathrm{a}, \mathrm{b}}$ \\
\hline $\begin{array}{l}\text { Proporção de exames citopatológicos sem citologia } \\
\text { anterior }\end{array}$ & $\begin{array}{l}\text { Número de exames citopatológicos sem relato de exame } \\
\text { anterior em mulheres de } 25 \text { a } 59 \text { anos x } 100 \text { / (número total } \\
\text { de exames nessa faixa etária - [número de exames nessa faixa } \\
\text { etária nos quais a mulher não soube informar + número de } \\
\text { exames nessa faixa etária sem essa informação]) }\end{array}$ & SISCOLO $^{\mathrm{a}, \mathrm{b}}$ \\
\hline $\begin{array}{l}\text { Proporção de exames citopatológicos com periodicidade } \\
\text { trienal }\end{array}$ & $\begin{array}{l}\text { Número de exames citopatológicos do colo do útero, em } \\
\text { mulheres de } 25 \text { a } 59 \text { anos, com relato de relato de exame } \\
\text { anterior há três anos ( } \leq 1 \text { ano, } 2 \text { anos e } 3 \text { anos) x } 100 \text { / total de } \\
\text { exames com relato de citopatologia anterior nessa faixa etária } \\
\text { - número de exames nessa faixa etária com essa informação } \\
\text { ignorada }\end{array}$ & SISCOLO $^{\mathrm{a}, \mathrm{b}}$ \\
\hline $\begin{array}{l}\text { Proporção de exames com citopatologia anterior a até } \\
\text { um ano }\end{array}$ & $\begin{array}{l}\text { Número de exames citopatológicos do colo do útero em } \\
\text { mulheres de } 25 \text { a } 59 \text { anos que relataram citopatologia anterior } \\
\text { em até um ano ( } \leq 1 \text { ano) x } 100 \text { / total de exames com relato de } \\
\text { citopatologia anterior nessa faixa etária - número de exames } \\
\text { com essa informação ignorada }\end{array}$ & SISCOLO $^{\mathrm{a}, \mathrm{b}}$ \\
\hline $\begin{array}{l}\text { Proporção de amostras insatisfatórias em exames } \\
\text { citopatológicos }\end{array}$ & $\begin{array}{l}\text { Número de exames citopatológicos } \\
\text { insatisfatórios x } 100 \text { / número total de exames }\end{array}$ & SISCOLO $^{\mathrm{a}, \mathrm{b}}$ \\
\hline $\begin{array}{l}\text { Proporção de exames com representatividade da Zona de } \\
\text { Transformação entre exames citopatológicos com amostra } \\
\text { satisfatória, por grupo etário ( }<50 \text { anos e } \geq 50 \text { anos) }\end{array}$ & $\begin{array}{l}\text { Número de exames citopatológicos com representatividade da } \\
\text { ZT x } 100 \text { / número total de exames com amostra satisfatória }\end{array}$ & SISCOLO $^{\mathrm{a}, \mathrm{b}}$ \\
\hline $\begin{array}{l}\text { Índice de positividade de exames citopatológicos do colo } \\
\text { do útero }\end{array}$ & $\begin{array}{l}\text { Número de exames citopatológicos com resultados alterados } \\
\text { x } 100 \text { / número de exames citopatológicos com amostra } \\
\text { satisfatória }\end{array}$ & SISCOLO $^{\mathrm{a}, \mathrm{b}}$ \\
\hline $\begin{array}{l}\text { Proporção dos tipos de alterações* por grupo etário } \\
\text { ( }<25 \text { anos, } 25 \text { a } 59 \text { anos e } \geq 60 \text { anos) }\end{array}$ & $\begin{array}{l}\text { Número de exames com determinada alteração (atipias, LSIL, } \\
\text { HSIL, carcinoma escamoso, adenocarcinoma e outros tipos) x } \\
100 \text { / total de exames alterados }\end{array}$ & SISCOLO $^{\mathrm{a}, \mathrm{b}}$ \\
\hline
\end{tabular}

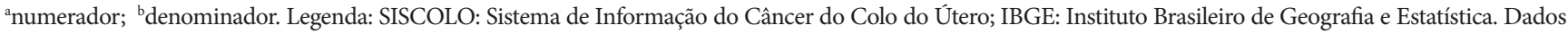
obtidos na página do DATASUS ${ }^{10}$. Nota: ${ }^{*}$ Alterações agrupadas nas categorias atipias de significado indeterminado (escamosa - possivelmente não neoplásica; escamosa - não se pode afastar lesão intraepitelial de alto grau; glandular - possivelmente não neoplásica; glandular - não se pode afastar lesão intraepitelial de alto grau; origem indefinida - possivelmente não neoplásica; origem indefinida - não se pode afastar lesão intraepitelial de alto grau); lesões de baixo grau (lesão intraepitelial de baixo grau - NIC I); lesões de alto grau (lesão intraepitelial de alto grau - NIC II e NIC III; lesão intraepitelial de alto grau não podendo afastar microinvasão); carcinoma escamoso (carcinoma epidermoide invasor); adenocarcinoma (adenocarcinoma in situ e adenocarcinoma invasor) e outras neoplasias

possível avaliar se a realização dos exames quanto à idade e frequência de repetição está de acordo com as recomendações do Ministério da Saúde ${ }^{13}$.

A qualidade do exame foi analisada pelos indicadores: percentual de amostras insatisfatórias nos exames citopatológicos do colo do útero, percentual de exames com representatividade da Zona de Transformação (ZT) e o índice de positividade. A análise da representatividade da ZT como indicador da qualidade da coleta deve-se ao fato de que é principalmente nessa área que surgem as lesões precursoras do CCU. A análise foi feita por faixa etária ( $<50$ anos e $\geq 50$ anos) a fim de diferenciar mulheres mais jovens daquelas na peri ou pós-menopausa, uma vez que estas últimas possuem maior probabilidade de apresentar mucosa vaginal atrófica, interferindo na coleta de uma amostra com representatividade da $\mathrm{ZT}^{14}$. O índice de positividade expressa a prevalência de alterações celulares nos exames e a sensibilidade do processo de rastreamento em detectar lesões na população examinada. Esse indicador pode ser categorizado em: muito baixo (<2\%), baixo ( 2 a $2,9 \%$ ), esperado (3 a $10 \%$ ) e acima do esperado $(>10 \%)^{15}$.

As alterações celulares no exame citopatológico do colo do útero foram agrupadas nas categorias: atipias de significado indeterminado; lesões de baixo grau; lesões de alto grau; carcinoma escamoso; adenocarcinoma e outras neoplasias. A fim de verificar o percentual de mulheres com lesão intraepitelial 
de alto grau (HSIL) que tiveram seu seguimento informado no SISCOLO, utilizou-se o relatório "consolidado de dados" disponível no SISCOLO.

Os dados brutos foram analisados de forma descritiva no programa Microsoft Excel 2007, por meio do cálculo dos indicadores expressos em razões e proporções.

Este estudo é parte de um projeto de pesquisa mais amplo, que objetivou analisar os resultados das ações previstas na Rede Viva Vida referentes ao rastreamento do CCU e de mama no período de 2006 a 2011 e foi aprovado pelo Comitê de Ética em Pesquisa da Universidade Federal de Juiz de Fora sob o Parecer no 1.376.660/2015.

\section{RESULTADOS}

Em 2011, a população de Minas Gerais era de 19.728.701 habitantes, dos quais $50,8 \%$ eram do sexo feminino, sendo $24,6 \%$ (4.846.569) mulheres de 25 a 59 anos. De 2006 a 2011, foram realizados 5.813.294 exames citopatológicos do colo do útero em mulheres nessa faixa etária no Estado.

No período analisado, observa-se que a razão de exames citopatológicos do colo do útero em mulheres de 25 a 59 anos manteve-se estável em Minas Gerais, porém sem alcançar a meta pactuada. Em 2008 e 2009, a meta foi de 0,30, igual ao parâmetro nacional, não tendo sido alcançada em nenhuma região. Norte, Nordeste e Jequitinhonha se destacaram superando a meta nos anos 2007, 2010 e 2011. No entanto, a Noroeste se destaca com o pior desempenho de 2007 a 2011 (Tabela 1).
A maior parte dos exames $(75,2 \%)$ foi realizada em mulheres de 25 a 59 anos, população-alvo do programa de rastreamento do CCU até 2011, porém observou-se um número considerável de exames (16\%) em mulheres com menos de 25 anos. De acordo com a Tabela 2, em Minas Gerais, houve progressiva redução na proporção de exames citopatológicos do colo do útero sem citologia anterior entre 2006 e 2011. Entre as macrorregiões, a Nordeste apresentou o maior percentual em 2006, e a Noroeste, de 2007 a 2011. Contudo, Centro-Sul e Triângulo do Norte tiveram os menores valores em 2006 e 2007, e a macrorregião Sul apresentou os menores nos de 2008 à 2011.

A maioria dos exames foi realizada dentro da periodicidade recomendada ( $\leq 3$ anos), com percentuais acima de $79 \% \mathrm{e}$ pouca diferença entre as macrorregiões. Em Minas Gerais, no período de análise, cerca da metade dos exames anteriores em mulheres de 25 a 59 anos foi realizada no período de até um ano: $48,8 \% ; 51,3 \%, 52,3 \% ; 50,9 \% ; 50,7 \%$ e $53 \%$, em 2006, 2007, 2008, 2009, 2010 e 2011, respectivamente. A macrorregião Triângulo do Sul apresentou os maiores percentuais em 2006 (55,1\%), 2008 (57,2\%) e 2009 (55,6\%); Sudeste, em 2007 (55,5\%); Triângulo do Norte, em 2010 (57,8\%); e Sul, em 2011 (60,3\%).

O percentual de amostras insatisfatórias em Minas Gerais ficou praticamente estável no período, em torno de $0,7 \%$. Os piores resultados foram observados na macrorregião Nordeste, que apresentou um considerável aumento no ano de 2009 (2,6\%). No mesmo ano, Jequitinhonha também se destacou com $2,5 \%$ de amostras insatisfatórias.

Tabela 1. Razão de exames citopatológicos em mulheres de 25 a 59 anos nas macrorregiões de saúde de Minas Gerais e número de exames e população feminina na faixa etária alvo do rastreamento em Minas Gerais, 2006-2011

\begin{tabular}{|c|c|c|c|c|c|c|}
\hline Macrorregiões & 2006 & 2007 & 2008 & 2009 & 2010 & 2011 \\
\hline Centro & 0,17 & 0,18 & 0,17 & 0,15 & 0,17 & 0,17 \\
\hline Centro-Sul & 0,22 & 0,19 & 0,19 & 0,19 & 0,20 & 0,21 \\
\hline Jequitinhonha & 0,25 & 0,26 & 0,23 & 0,24 & 0,28 & 0,28 \\
\hline Leste & 0,19 & 0,21 & 0,20 & 0,21 & 0,23 & 0,22 \\
\hline Leste do Sul & 0,22 & 0,21 & 0,21 & 0,21 & 0,22 & 0,26 \\
\hline Nordeste & 0,25 & 0,25 & 0,22 & 0,24 & 0,28 & 0,26 \\
\hline Noroeste & 0,23 & 0,11 & 0,10 & 0,10 & 0,10 & 0,11 \\
\hline Norte & 0,26 & 0,28 & 0,26 & 0,27 & 0,25 & 0,28 \\
\hline Oeste & 0,21 & 0,21 & 0,22 & 0,22 & 0,22 & 0,24 \\
\hline Sudeste & 0,23 & 0,21 & 0,21 & 0,21 & 0,20 & 0,20 \\
\hline Sul & 0,22 & 0,22 & 0,23 & 0,24 & 0,24 & 0,25 \\
\hline Triângulo do Norte & 0,21 & 0,21 & 0,22 & 0,22 & 0,23 & 0,24 \\
\hline Triângulo do Sul & 0,22 & 0,24 & 0,21 & 0,20 & 0,22 & 0,21 \\
\hline \multicolumn{7}{|l|}{ Minas Gerais } \\
\hline Exames & 868.279 & 970.519 & 951.677 & 966.032 & 1.009 .463 & 1.047 .324 \\
\hline População & 4.247 .165 & 4.798 .458 & 4.886 .044 & 4.981 .484 & 5.002 .386 & 5.035 .579 \\
\hline Razão & 0,20 & 0,21 & 0,20 & 0,20 & 0,21 & 0,22 \\
\hline Meta - SISPACTO & -- & 0,23 & 0,30 & 0,30 & 0,22 & 0,25 \\
\hline
\end{tabular}

Fonte: Sistema de Informação do Câncer do Colo do Útero (SISCOLO $)^{10}$ 
Tabela 2. Proporção de exames citopatológicos do colo do útero sem citologia anterior em mulheres de 25 a 59 anos, por ano, em Minas Gerais e suas macrorregiões de saúde. Minas Gerais, 2006-2011

\begin{tabular}{lrrrrrc}
\multicolumn{1}{c}{ Macrorregiões } & $\mathbf{2 0 0 6}$ & $\mathbf{2 0 0 7}$ & $\mathbf{2 0 0 8}$ & $\mathbf{2 0 0 9}$ & $\mathbf{2 0 1 0}$ & $\mathbf{2 0 1 1}$ \\
Centro & 10,0 & 10,0 & 6,6 & 5,1 & 4,0 & 3,3 \\
Centro-Sul & 8,0 & 7,6 & 6,0 & 5,4 & 5,0 & 4,3 \\
Jequitinhonha & 14,0 & 10,5 & 7,8 & 7,6 & 7,7 & 7,1 \\
Leste & 11,6 & 9,6 & 8,2 & 6,6 & 6,7 & 5,7 \\
Leste do Sul & 13,8 & 10,2 & 8,9 & 8,9 & 8,5 & 7,4 \\
Nordeste & 20,7 & 12,5 & 10,1 & 7,8 & 6,9 & 6,5 \\
Noroeste & 19,9 & 18,7 & 41,8 & 34,2 & 9,6 & 17,7 \\
Norte & 16,4 & 14,7 & 13,8 & 5,7 & 4,8 & 4,3 \\
Oeste & 12,2 & 6,3 & 5,0 & 4,7 & 4,1 & 3,6 \\
Sudeste & 12,6 & 6,1 & 8,4 & 7,0 & 4,9 & 4,5 \\
Sul & 10,3 & 5,2 & 4,3 & 2,7 & 3,0 & 3,3 \\
Triângulo do Norte & 8,0 & 4,3 & 5,0 & 5,5 & 5,5 & 4,5 \\
Triângulo do Sul & 9,7 & 6,4 & 6,0 & 7,0 & 7,1 & 5,1 \\
\hline \multicolumn{1}{c}{ MINAS GERAIS } & & & & & & \\
n & 77222 & 77866 & 68090 & 52774 & 43553 & 42346 \\
\% & 12,0 & 9,1 & 8,4 & 6,4 & 5,0 & 4,7 \\
\hline
\end{tabular}

Fonte: Sistema de Informação do Câncer do Colo do Útero (SISCOLO) ${ }^{10}$

Tabela 3. Índice de positividade de exames citopatológicos do colo do útero, segundo ano, em Minas Gerais e suas macrorregiões de saúde. Minas Gerais, 2006 a 2011

\begin{tabular}{lcccccc}
\multicolumn{1}{c}{ Macrorregiões } & $\mathbf{2 0 0 6}$ & $\mathbf{2 0 0 7}$ & $\mathbf{2 0 0 8}$ & $\mathbf{2 0 0 9}$ & $\mathbf{2 0 1 0}$ & $\mathbf{2 0 1 1}$ \\
Centro & 1,9 & 2,7 & 2,2 & 2,3 & 2,2 & 3,1 \\
Centro-Sul & 1,5 & 2,2 & 1,7 & 1,5 & 1,3 & 1,4 \\
Jequitinhonha & 1,2 & 1,3 & 1,0 & 0,8 & 1,2 & 1,2 \\
Leste & 3,3 & 2,8 & 2,0 & 2,0 & 2,6 & 3,6 \\
Leste do Sul & 1,5 & 2,0 & 1,7 & 1,5 & 1,5 & 1,5 \\
Nordeste & 2,0 & 2,6 & 2,1 & 1,6 & 1,4 & 2,8 \\
Noroeste & 1,9 & 2,7 & 2,0 & 1,6 & 1,6 & 2,3 \\
Norte & 1,8 & 2,2 & 1,6 & 1,4 & 1,6 & 2,6 \\
Oeste & 1,1 & 1,7 & 1,3 & 1,3 & 1,7 & 2,2 \\
Sudeste & 2,2 & 4,8 & 2,8 & 2,5 & 2,8 & 3,5 \\
Sul & 3,0 & 3,4 & 2,5 & 2,4 & 2,8 & 4,0 \\
Triângulo do Norte & 1,7 & 3,0 & 2,0 & 1,5 & 1,6 & 2,5 \\
Triângulo do Sul & 3,9 & 5,8 & 4,4 & 5,2 & 6,0 & 7,6 \\
MINAS GERAIS & 2,2 & 2,2 & 2,2 & 2,1 & 2,3 & 2,3 \\
\hline Fonte: Sistema de Informação do Câncer do Colo do Útero (SISCOLO) ${ }^{10}$
\end{tabular}

No que se refere à representatividade da ZT em exames citopatológicos do colo do útero, em 2006, Minas Gerais obteve o pior resultado: $46,9 \%$ para a faixa etária inferior a 50 anos e $37,2 \%$ para mulheres com 50 anos ou mais. Em 2007 e 2008, apresentou os melhores desempenhos do período: $72,9 \%$ para a faixa etária inferior a 50 anos e $55,2 \%$ para mulheres com 50 anos ou mais e $71,1 \%$ para a faixa etária inferior a 50 anos e $52,8 \%$ para mulheres com 50 anos ou mais, respectivamente. Quanto às macrorregiões, a Norte foi a que apresentou o maior percentual de amostras com representatividade ZT nas duas faixas etárias analisadas, com percentuais que variaram de $56,2 \%$, em 2006 , para a faixa etária igual ou superior a 50 anos, a 93,7\%, em 2007, para a faixa etária inferior a 50 anos. Triângulo do Norte e Leste do Sul apresentaram os piores percentuais em mulheres com menos de 50 anos (Triângulo do Norte no período 2006-2009: $28,1 \% ; 44,6 \%$; $43,5 \%$ e $42,8 \%$, respectivamente; e Leste do Sul no período 2010-2011: 43,3\% e 39,9\%, respectivamente), e com 50 anos ou mais (Triângulo do Norte nos anos 2006 e 2011: $21,9 \%$ e $31,7 \%$, respectivamente; e Leste do Sul no período 2007-2010: 33,6\%; 33,6\%; $29 \%$ e $28,5 \%$, respectivamente). Em 2006, destacou-se a macrorregião Triângulo do Norte, que apresentou representatividade da ZT em apenas 28,1 e 21,9\% dos exames realizados em mulheres com menos de 50 anos ou 50 anos e mais, respectivamente.

Na Tabela 3, observa-se que o índice de positividade no Estado durante todo o período de análise foi muito baixo $(<2 \%)$. Triângulo do Sul foi a única macrorregião que apresentou categorização do índice de positividade dentro do esperado (3-10\%), de 2006 a 2011. Jequitinhonha e Leste do Sul, no entanto, apresentaram percentuais abaixo do esperado em todos os anos. A macrorregião Centro destacou-se com uma gradativa melhora no período.

As atipias foram os principais tipos de alterações em Minas Gerais no período de 2006 a 2011, nas faixas etárias de 25 a 59 anos e $\geq 60$ anos, representando $54,2 \%$ e $60,3 \%$ das alterações, respectivamente. Observou-se que o percentual de atipias cresceu com o aumento da faixa etária: em 2006, representou 38\% das alterações em mulheres com menos de 25 anos, 50,5\% das alterações em mulheres de 25 a 59 anos e $56,7 \%$ na faixa etária igual ou maior a 60 anos. Em 2011, representou $48,7 \%$; $56,2 \%$ e $59,4 \%$, respectivamente. Nas mulheres com menos de 25 anos, as lesões intraepiteliais de baixo grau LSIL corresponderam a 51,4\% das alterações nessa faixa etária no período estudado, sendo as mais frequentes, exceto no ano de 2011, no qual as atipias foram o tipo de lesão mais frequente (atipias: $48,7 \%$; LSIL: $48 \%$ ).

As HSIL foram mais frequentes em mulheres com 60 anos ou mais, com uma média de 19,3\% das alterações. Carcinoma escamoso, adenocarcinoma e outros tipos de neoplasias também foram mais frequentes nessa faixa etária, representando, respectivamente, 4,6, 1,6 e 0,4\% das alterações. Além disso, verificou-se, nessa faixa etária, redução no percentual de carcinoma escamoso ao final do período analisado - $6,4 \% \mathrm{em}$ 2006 e 2,9\% em 2011.

Como pode ser observado na Tabela 4, em Minas Gerais, o percentual de seguimento informado de mulheres com lesões intraepiteliais de alto grau (HSIL) ficou em torno de 55\%. A macrorregião Triângulo do Norte se destacou em 2006, com apenas 9,4\% de seguimento informado, e, de 2007 a 2011, a Sudeste apresentou os piores resultados para esse indicador. 
Tabela 4. Proporção de seguimento informado de mulheres com lesão intraepitelial de alto grau do colo do útero, segundo o ano de diagnóstico, em Minas Gerais e suas macrorregiões de saúde. Minas Gerais, 2006-2011

\begin{tabular}{lcccccc}
\multicolumn{1}{c}{ Macrorregiões } & $\mathbf{2 0 0 6}$ & $\mathbf{2 0 0 7}$ & $\mathbf{2 0 0 8}$ & $\mathbf{2 0 0 9}$ & $\mathbf{2 0 1 0}$ & $\mathbf{2 0 1 1}$ \\
Centro & 55,9 & 62,2 & 71,3 & 79,7 & 83,0 & 73,6 \\
Centro-Sul & 63,5 & 82,3 & 95,6 & 90,8 & 82,1 & 49,4 \\
Jequitinhonha & 66,7 & 84,6 & 62,5 & 69,2 & 57,9 & 38,9 \\
Leste & 47,6 & 36,8 & 57,3 & 83,8 & 63,8 & 49,3 \\
Leste do Sul & 70,6 & 80,0 & 94,7 & 85,7 & 88,1 & 91,7 \\
Nordeste & 43,4 & 44,2 & 59,1 & 60,0 & 60,6 & 38,1 \\
Noroeste & 40,0 & 53,5 & 46,0 & 47,5 & 46,8 & 48,1 \\
Norte & 26,2 & 30,4 & 53,2 & 44,7 & 40,5 & 31,8 \\
Oeste & 73,4 & 78,2 & 59,8 & 69,0 & 53,3 & 35,2 \\
Sudeste & 29,5 & 20,6 & 22,0 & 28,4 & 27,9 & 18,1 \\
Sul & 76,5 & 71,8 & 88,2 & 84,6 & 83,3 & 80,6 \\
Triângulo do & 9,3 & 23,2 & 74,8 & 72,2 & 61,3 & 56,1 \\
Norte & & & & & & \\
Triângulo do Sul & 37,0 & 29,6 & 43,0 & 40,6 & 45,7 & 53,2 \\
MINAS GERAIS & 48,7 & 47,6 & 57,5 & 62,4 & 59,4 & 52,7 \\
\hline
\end{tabular}

Fonte: Sistema de Informação do Câncer do Colo do Útero (SISCOLO) ${ }^{10}$

\section{DISCUSSÃO}

Em Minas Gerais, no período de 2006 a 2011, observou-se estabilidade na razão de exames citopatológicos em mulheres de 25 a 59 anos, não tendo atingido a meta pactuada, o que sugere baixo acesso da população-alvo ao programa de rastreamento do CCU. A maior parte dos exames foi realizada na população-alvo, e houve progressiva redução na proporção de citopatológicos do colo do útero sem citologia anterior. A maioria dos exames ocorreu dentro da periodicidade recomendada, dos quais cerca da metade no período de até um ano. Quanto à qualidade do exame, destaca-se que o índice de positividade foi categorizado como baixo ao longo de todo o período analisado. Além disso, observou-se baixo percentual de seguimento informado no Estado.

Minas Gerais se caracteriza pela grande disparidade socioeconômica entre suas macrorregiões de saúde, destacando-se dois blocos quanto ao valor do Produto Interno Bruto (PIB) per capita referente a 2011: por um lado, as macrorregiões Nordeste, Jequitinhonha, Norte e Leste do Sul com os menores valores e, por outro lado, Triângulo do Norte, Centro, Triângulo do Sul e Sul com os maiores valores.

Jequitinhonha, Nordeste e Norte, de menor nível socioeconômico, destacaram-se com os altos valores de razão de exames na população estudada, ultrapassando a meta estadual em 2007, 2010 e 2011. A razão de exames em mulheres de 25 a 59 anos é considerada um indicador de desempenho da Atenção Primária à Saúde (APS $)^{16}$. Fontanive e colaboradores ${ }^{17}$ verificaram que municípios com maior cobertura da Estratégia Saúde da Família (ESF) no Rio Grande do Sul tiveram maior razão de citopatológicos na população-alvo. Dessa forma, representa um importante preditor de realização de exames citopatológicos, o que pode ser atribuído ao vínculo profissional-paciente fortalecido nesse nível devido à longitudinalidade da assistência. Além disso, a territorialização e o cadastramento das famílias pela ESF favorece a identificação de mulheres que nunca fizeram o exame citológico ou que estão em atraso ${ }^{7}$. Dentro desse contexto, Tomasi e colaboradores ${ }^{18}$ sugerem que a adoção da ESF como modelo de atenção básica parece contribuir para a melhoria da estrutura dos serviços de saúde e, consequentemente, para a prestação de serviços voltados à prevenção do câncer de colo uterino.

Estudo ecológico com base em dados secundários de 2008 a 2012 em Minas Gerais verificou maior cobertura de ESF em microrregiões de saúde do Estado com alta vulnerabilidade em saúde e baixo nível socioeconômico (Norte, Noroeste, Nordeste, Jequitinhonha e Leste) ${ }^{19}$. Assim, as altas razões de exames na população-alvo nas regiões Jequitinhonha, Nordeste e Norte observadas no presente estudo podem estar relacionadas à maior cobertura pela ESF. Contudo, a região Noroeste, apesar de possuir alta cobertura de ESF, apresentou resultados desfavoráveis, o que pode estar relacionado à rotatividade de profissionais nessas equipes, interferindo negativamente no vínculo construído com as famílias, à dificuldade de acesso das usuárias ao SUS para a coleta do exame citopatológico ou às barreiras das próprias mulheres em relação ao exame de Papanicolau ${ }^{20}$.

Em relação a esse indicador, deve-se ressaltar que a razão de exames citopatológicos não mensura diretamente a cobertura, já que avalia a oferta de Papanicolau com base no número de exames, e não de mulheres examinadas. Além disso, pode estar subestimado em regiões com alta cobertura pela saúde suplementar, pois considera apenas os exames realizados pelo SUS ${ }^{21}$.

Em outro estudo, também realizado utilizando dados do SISCOLO para Minas Gerais, referentes ao período 2000-2010, verificou-se melhora do percentual da meta alcançada do rastreamento do CCU em mulheres de 25 a 59 anos em 2010 quando comparada ao ano de 2000 (63,8\% em 2000 e 89,3\% em 2010), observando os melhores resultados nas macrorregiões Nordeste, Jequitinhonha e Sul20.

A maioria dos exames em Minas Gerais foi realizada em mulheres na faixa etária preconizada pelo Ministério da Saúde, porém observou-se percentual considerável de exames em mulheres menores de 25 anos. Tal resultado confirma os achados de um estudo ecológico que também utilizou dados do SISCOLO para o Brasil e regiões no período de 2006 a $2009^{21}$. No presente estudo, de 2006 a 2011, observou-se uma média anual de 206.430 exames em mulheres com menos de 25 anos. Se esses exames tivessem sido realizados na faixa etária preconizada, a razão de exames seria: 0,25 em 2006, 2007 e 2011 e 0,24 em 2008, 
2009 e 2010, o que permitiria o alcance da meta estadual nos anos 2007, 2010 e 2011.

Em relação à periodicidade de realização, assim como observado para o Brasil, a maioria dos exames foi repetida em até um ano, sugerindo o não cumprimento das recomendações nacionais ${ }^{15}$. De acordo com o INCA ${ }^{5}$, apenas as mulheres que iniciaram o rastreamento, as que tiveram exames com amostra insatisfatória e as que apresentaram alterações com necessidade de controle em intervalo menor deveriam repetir o exame no período de um ano. Nesse contexto, o percentual de exames com periodicidade anual talvez seja superior às recomendações nesse prazo $^{22}$. Dessa forma, a cobertura da população-alvo é provavelmente menor que o ideal, com as mesmas mulheres repetindo anualmente o citopatológico, enquanto outras continuam sem acesso ${ }^{23}$.

Nessa perspectiva, considera-se que exames em excesso correspondem a todos aqueles realizados fora do grupo etário alvo ou àqueles com intervalo desde o último controle menor do que três anos. Estudo realizado no município de Amparo/SP encontrou percentuais de exames em excesso que variaram entre $61,2 \%$ em 2002 e 65,5\% em 2005, o que correspondeu a quase dois terços do total dos exames realizados ${ }^{7}$. Quanto à faixa etária, cabe ressaltar que abaixo dos 25 anos predominam as LSIL, que regridem espontaneamente na maioria dos casos, e que a repetição anual do exame não aumenta significativamente o efeito protetor do rastreamento. A realização de exames fora da faixa etária alvo e da periodicidade recomendada, além de sobrecarregar os serviços, pode comprometer o acesso das mulheres que realmente precisam ser rastreadas e encaminhadas para a investigação diagnóstica e tratamento das lesões precursoras ${ }^{23}$.

Observou-se diminuição progressiva de exames sem citologia anterior em Minas Gerais ao longo do período estudado. De acordo com o INCA ${ }^{23}$, em geral, há um decréscimo no percentual de exames em mulheres que nunca o realizaram, o que pode indicar que o programa aumentou a cobertura ao longo do tempo e, com isso, diminuiu a captação de mulheres sem citologia anterior ${ }^{21}$. Porém, tal indicador deve ser visto com cautela, pois é esperado que, a cada ano, novas mulheres iniciem o rastreamento ao entrar na faixa etária alvo. Como no presente estudo observaram-se estabilidade da oferta de exames e alta proporção de exames com repetição anual, esses resultados podem refletir oferta inadequada de exames e menor capacidade para captação de mulheres que nunca realizaram o exame ${ }^{24}$.

Em relação à qualidade do exame, o limite para o percentual de exames insatisfatórios é de 5\%, mas a meta deve tender a zero para garantir a efetividade da ação do rastreamento ${ }^{15}$. A análise desse indicador para Minas Gerais mostrou relativa estabilidade no período, com resultados satisfatórios, sempre abaixo de 1\%. Nordeste e Jequitinhonha se destacaram com os piores percentuais, especialmente em 2009. Para o Brasil, estudo realizado com dados referentes ao período de 2002 a 2006 verificou que o percentual de amostras insatisfatórias se manteve em torno de $1 \%{ }^{22}$.

Em Minas Gerais, o indicador representatividade da ZT apresentou o pior resultado em 2006, com melhora nos anos seguintes. Triângulo do Norte e Leste do Sul chamaram a atenção com resultados insatisfatórios, o que pode levar ao subdiagnóstico de alterações. Nesse sentido, a baixa qualidade observada pelos indicadores de qualidade do exame, especialmente nas macrorregiões Nordeste, Jequitinhonha, Triângulo do Norte e Leste do Sul, indica a necessidade de qualificação dos profissionais para a coleta do exame citopatológico do colo do útero ${ }^{14}$.

O índice de positividade de exames de Papanicolau no Estado foi baixo ao longo de todo o período analisado, semelhante ao encontrado para o Brasil (2,5\%) para o período de análise de 2002 a $2011^{15}$. Jequitinhonha e Leste do Sul se destacaram com resultados muito baixos para esse indicador, o que pode sugerir que alterações suspeitas não estão sendo identificadas, resultando em exames falso-negativos. Nesse sentido, a avaliação da qualidade do exame citopatológico é fundamental, pois a coleta adequada possibilita diagnosticar correta e precocemente as lesões precursoras, reduzindo os resultados falso-negativos e a necessidade de repetições de exames. Assim, a qualidade do exame está diretamente relacionada ao sucesso do rastreamento do $\mathrm{CCU}^{25}$.

Os resultados do estudo revelaram que os principais tipos de alterações no Estado foram as atipias, constatando-se aumento dos percentuais com a elevação da faixa etária. Dessa forma, destaca-se que, em mulheres com 60 anos ou mais, as atipias corresponderam a $60,3 \%$ do total de alterações no período analisado. De forma geral, na faixa etária inferior a 25 anos, houve predominância de LSIL. As HSIL foram mais frequentes na faixa etária igual ou maior a 60 anos. Resultados semelhantes foram verificados em estudo com base em dados do SISCOLO em Teresina, no Piauí, no período de 2006-2013²4. Dentro desse contexto, destaca-se que o percentual de atipias indica indiretamente a qualidade da coleta e da análise laboratorial e aponta, quando predominante, para a necessidade de melhoria na qualidade dos exames ${ }^{26}$.

No presente estudo, observou-se redução no percentual de carcinoma escamoso ao final do período analisado, assim como verificado por Dias e colaboradores para o Brasil, no período de 2002 a 2006, o que pode indicar aumento da resolutividade do Programa de Rastreamento de Câncer de Colo do Útero ${ }^{22}$.

Verificou-se que o percentual de seguimento para mulheres diagnosticadas com HSIL em 2010 com seguimento em 2011 foi baixo no Estado (52,7\%), mas bem superior ao verificado 
em âmbito nacional, que foi de $21,5 \%{ }^{14}$. Isso pode indicar que a informação de seguimento não está sendo registrada ou que mulheres com HSIL não estão sendo acompanhadas. O objetivo do rastreamento é identificar e tratar precocemente as lesões precursoras. Assim, a informação do seguimento das mulheres que tiveram esse diagnóstico é fundamental para avaliar as ações do programa do controle do $\mathrm{CCU}^{13}$.

Para que haja uma efetiva redução da incidência e da mortalidade pelo CCU, é necessário que o programa de rastreamento seja ofertado àquelas mulheres pertencentes aos grupos de risco preconizados por meio de exames de qualidade e que o tratamento adequado seja propiciado às mulheres que apresentam resultados positivos ${ }^{27}$.

Os dados do SISCOLO permitiram a construção de indicadores fundamentais para avaliar as ações do rastreamento do CCU em Minas Gerais, podendo subsidiar o planejamento das ações de saúde. Com a implantação do SISCAN (Sistema de Informação do Câncer), que substitui e integra o SISCOLO e o SISMAMA (Sistema de Informação do Câncer de Mama), algumas limitações desses sistemas serão superadas e permitirão o acompanhamento longitudinal das usuárias, pois o registro terá como unidade de observação a mulher, e não o exame.

Diante dos achados, percebe-se que o programa de rastreamento do CCU em Minas Gerais apresenta limitações, podendo-se verificar as diferenças regionais em relação aos indicadores analisados. Os resultados evidenciam a necessidade de aprimoramento do programa de rastreamento em relação à oferta e qualidade do exame, bem como ao direcionamento correto quanto à faixa etária e à periodicidade, visando garantir o acesso da população-alvo e encaminhamento para a investigação diagnóstica e tratamento das lesões precursoras, quando indicado.

\section{REFERÊNCIAS}

1. International Agency for Research on Cancer. Globocan 2012 [Internet]. Lyon: IARC; 2012 [citado em 2016 set 23]. Disponível em: http://globocan. iarc.fr/old/FactSheets/cancers/cervix-new.asp

2. World Health Organization. Comprehensive cervical cancer control: a guide to essential practice. 2 ed. Geneva: WHO; 2014.

3. Instituto Nacional de Câncer José Alencar Gomes da Silva. Coordenação de Prevenção e Vigilância. Estimativa 2016: incidência de câncer no Brasil [Internet]. Rio de Janeiro: INCA; 2015 [citado em 2016 jun 7]. Disponível em: http://www.inca.gov.br/estimativa/2016/estimativa-2016-v11.pdf

4. Girianelli VR, Gamarra CJ, Azevedo e Silva G. Os grandes contrastes na mortalidade por câncer do colo uterino e de mama no Brasil. Rev Saude Publica. 2014;48(3):459-67. PMid:25119941. http://dx.doi.org/10.1590/ S0034-8910.2014048005214.

5. Instituto Nacional de Câncer José Alencar Gomes da Silva. Coordenação de Prevenção e Vigilância. Divisão de Deteç̧ão Precoce e Apoio à Organização de Rede. Diretrizes brasileiras para o rastreamento do câncer do colo do útero [Internet]. Rio de Janeiro: INCA; 2016 [citado em 2016 jun 1]. Disponível em: http://www2.inca.gov.br/wps/wcm/connect/9000f2004b3 9c00db985bf66c974cd7f/Diretrizes+Brasileiras +2016 vers\%C3\%A3o+C onsulta + P\%C3\%BAblica.pdf?MOD=AJPERES\&CACHEID $=9000 \mathrm{f} 2004 \mathrm{~b}$ $39 \mathrm{c} 00 \mathrm{db} 985 \mathrm{bf} 66 \mathrm{c} 974 \mathrm{~cd} 7 \mathrm{f}$

6. World Health Organization. National cancer control programmes: policies and managerial guidelines. 2 ed. Geneva: WHO; 2002.

7. Vale DBAP, Morais SS, Pimenta AL, Zeferino LC. Avaliação do rastreamento do câncer do colo do útero na Estratégia Saúde da Família no Município de Amparo, São Paulo, Brasil. Cad Saude Publica. 2010;26(2):383-90. PMid:20396853. http://dx.doi.org/10.1590/S0102-311X2010000200017.

8. Brasil. Ministério da Saúde. Secretaria de Atenção à Saúde. Departamento de Atenção Básica. Rastreamento [Internet]. Brasília: Ministério da Saúde; 2010 [citado em 2016 jul 5]. (Série A. Normas e Manuais Técnicos. Cadernos de Atenção Primária, 29). Disponível em: http://bvsms.saude.gov.br/bvs/ publicacoes/caderno_atencao_primaria_29_rastreamento.pdf

9. Domingues EP, Magalhães AS, Faria WR. Infraestrutura, crescimento e desigualdade regional: uma projeção dos impactos dos investimentos do

Programa de Aceleração do Crescimento (PAC) em Minas Gerais. PPE. 2009;39(1):121-58

10. Brasil. Ministério da Saúde. Departamento de Informática do Sistema Único de Saúde [Internet]. 2016 [citado em 2016 ago 2]. Disponível em: http://datasus.saude.gov.br/

11. Brasil. Ministério da Saúde. Departamento de Apoio à Gestão Descentralizada. Instrutivo da Pactuação de Prioridades, Objetivos, Metas e Indicadores de Monitoramento do Pacto pela Vida e de Gestão para o Biênio 2010-2011. Conforme Portaria n 2669 GM/MS de 03 de novembro de 2009, Brasília: Ministério da Saúde; 2009

12. Instituto Nacional de Câncer José Alencar Gomes da Silva. Nomenclatura brasileira para laudos cervicais e condutas preconizadas - recomendações para profissionais de saúde. Revista Brasileira de Cancerologia, v. 52, n. 3 , p. 213-236, 2006.

13. Instituto Nacional de Câncer José Alencar Gomes da Silva. Coordenação Geral de Prevenção e Vigilância. Divisão de Detecção Precoce e Apoio à Organização de Rede. Ficha técnica de indicadores das ações de controle do câncer do colo do útero [Internet]. Rio de Janeiro: INCA; 2014 [citado em 2016 jun 25]. Disponível em: http://www2.inca.gov.br/wps/wcm/con nect/84f26080469faa79859bed5120665fa8/FICHA+T\%C3\%89CNICA+I ndicadores+Colo+14.pdf?MOD=AJPERES\&CACHEID $=84 \mathrm{f} 26080469 \mathrm{fa}$ a79859bed5120665fa8

14. Instituto Nacional de Câncer José Alencar Gomes da Silva. Monitoramento das ações de controle dos cânceres do colo do útero e de mama [Internet]. Informativo Detecção Precoce. 2012 jan/mar;3(1):1-8. [citado em 2016 jun 19]. Disponível em: http://wwwl.inca.gov.br/inca/Arquivos/informativo_ detecprecoce_1.2_2012.pdf

15. Instituto Nacional de Câncer José Alencar Gomes da Silva. Monitoramento das ações de controle dos cânceres do colo do útero e de mama [Internet]. Informativo Detecção Precoce. 2012 ago/dez;3(3):1-12. [citado em 2016 jun 20]. Disponível em: http://bvsms.saude.gov.br/bvs/publicacoes/inca/ Informativo_Deteccao_Precoce_agosto_dezembro_2012.pdf

16. Sala A, Mendes JDV. Perfil de Indicadores da Atenção Primária à Saúde no Estado de São Paulo: retrospectiva de 10 anos. Saude Soc. 2011;20(4):91226. http://dx.doi.org/10.1590/S0104-12902011000400009. 
17. Fontanive PV, Kolling JHG, Castro Fo ED, Harzheim E. Cobertura da Estratégia Saúde da Família e de citopatologia de colo uterino no Rio Grande do Sul. Rev Bras Med Fam e Com. 2008;4(14):119-28. http://dx.doi. org/10.5712/rbmfc4(14)194.

18. Tomasi E, Oliveira TF, Fernandes PAA, Thumé E, Silveira DS, Siqueira FV, et al. Estrutura e processo de trabalho na prevenção do câncer de colo de útero na Atenção Básica à Saúde no Brasil: Programa de Melhoria do Acesso e da Qualidade - PMAQ. Rev Bras Saude Mater Infant. 2015;15(2):171-80. http://dx.doi.org/10.1590/S1519-38292015000200003.

19. Duarte DAP. Iniquidade social e câncer em mulheres: análise da mortalidade por câncer de mama e colo do útero nas microrregiões de saúde de Minas Gerais no período de 2008-2012 [dissertação]. Juiz de Fora: Universidade Federal de Juiz de Fora; 2016

20. Nascimento GWC, Pereira CCA, Nascimento DIC, Lourenço GC, Machado CJ. Cobertura do exame citopatológico do colo do útero no Estado de Minas Gerais, Brasil, no período entre 2000-2010: um estudo a partir dos dados do Sistema de Informação do câncer do Colo do Útero (SISCOLO). Cad Saude Colet. 2015;23(3):253-60. http://dx.doi.org/10.1590/1414$462 X 201500030059$.

21. Santos RS, Melo ECP, Santos KM. Análise espacial dos indicadorespactuados para o rastreamento do câncer do colo do útero no Brasil. Texto Contexto Enferm. 2012;21(4):800-10. http://dx.doi.org/10.1590/S0104-07072012000400010.

22. Dias MBK, Tomazelli JG, Assis M. Rastreamento do câncer de colo do útero no Brasil: análise de dados do Siscolo no período de 2002 a 2006. Epidemiol Serv Saúde. 2010;19(3):293-306.
23. Instituto Nacional de Câncer José Alencar Gomes da Silva. Monitoramento das ações de controle dos cânceres do colo do útero e de mama [Internet]. Informativo Detecção Precoce. 2014 jan/abr;5(1):1-8. [citado em 2016 jun 20]. Disponível em: http://www1.inca.gov.br/inca/Arquivos/inform_detec_ precoce_1_2014.pdf

24. Damacena AM, Luz LL, Mattos IE. Rastreamento do câncer do colo do útero em Teresina, Piauí: estudo avaliativo dos dados do Sistema de Informação do Câncer do Colo do Útero, 2006-2013. Epidemiol Serv Saúde. 2017;26(1):71-80.

25. Instituto Nacional de Câncer José Alencar Gomes da Silva. Monitoramento das ações de controle dos cânceres do colo do útero e de mama [Internet]. Informativo Deteç̧ão Precoce. 2015 jan/abr;6(1):1-8. [citado em 2016 ago 2]. Disponível em: http://www1.inca.gov.br/inca/Arquivos/comunicacao/ deteccao_precoce_12015.pdf

26. Bortolon PC, Silva MAF, Corrêa FM, Dias MBK, Knupp VMAO, Assis $\mathrm{M}$, et al. Avaliação da qualidade dos laboratórios de citopatologia do colo do útero no Brasil. Rev Bras Cancerol. 2012;58(3):435-44.

27. Martínez-Mesa J, Werutsky G, Campani RB, Wehrmeister FC, Barrios $\mathrm{CH}$. Inequalities in Pap smear screening for cervical cancer in Brazil. Prev Med. 2013;57(4):366-71. PMid:23827721. http://dx.doi.org/10.1016/j. ypmed.2013.06.026.

Recebido em: Jul. 15, 2017 Aprovado em: Set. 18, 2017 